\title{
The future of surgical robotics
}

\author{
Laura Callan, MASc (Meds 2015) and Nancy Chen, BSc (Meds 2016) \\ Faculty Reviewer: Dr. Stephen Pautler, MD, FRCSC (Department of Surgery, Division of Urology)
}

$\mathrm{R}$

obotic surgery is a growing technology with the potential to revolutionize surgical procedures, especially in the field of urology. Urology is at the forefront of advances in this field, and has integrated robotics into many procedures including radical cystectomies, surgical nerve grafting and pyeloplasty. ${ }^{1}$ Robotic surgery has almost entirely taken over radical prostatectomy and the role of robotics is continuously expanding. Robotic surgery helps improve patient outcome by minimizing the surgeon's movement tremors, increasing range of motion, decreasing blood loss, decreasing length of hospital stay, and decreasing post-operative pain. ${ }^{2,3}$ Since the field of Urology deals with very difficult and delicate procedures, robotics offers an advantage by allowing for greater accuracy, flexibility, smoother actions, and greater range of motion.

An important area of advancement for surgical robotics is in the field of imaging and visual feedback. Robotic surgery can be performed either with a surgeon controlling the robot such as is done with the da Vinci system to perform robotic-assisted radical prostatectomies (RARPs), or autonomously based on a predetermined algorithm such as in the case of radiosurgery for pituitary adenomas. ${ }^{4}$ While autonomous robotic systems offer higher precision, and tissue-damage avoidance, surgeon-controlled systems are much better at decision making in unstructured or chaotic situations. For the surgeon controlling the robot, visual feedback can be attained using endoscopic cameras; however, for autonomous procedures, the control program requires much more detail about the patient's anatomy.

Generally speaking, there are two types of imaging performed for robotic surgeries: pre-operative and intra-operative. Pre-operative imaging is acquired before a procedure and used for surgical planning to locate pathologies, a feed-forward system. In computer-guided systems this can be problematic since the images which are used for instrument navigation are based on tissue location at the time of imaging. However, instrument deflection caused by instrument-tissue interaction as well as tissue movement can mean that the instrument position is not as precise as is desirable. This is especially problematic when dealing with smaller anatomical targets, such as vascular surgery and in paediatrics. ${ }^{5}$ Intraoperative imaging is used to provide close to real-time feedback, thereby closing the feedback loop and allowing for adjustments throughout the procedure. While for surgeon controlled robotics, surgeons have the flexibility of compensating via visual feed-back, for autonomous computer-controlled systems this cannot be as easily implemented due to the need for code to interpret the visual feedback into a control for the robot.

Research into methods of providing a closed-loop feedback system which takes into account instrument deflection and tissue movement has been carried out using various approaches. Methods of measuring instrument position include computer vision techniques, ${ }^{6}$ and electromagnetic sensor systems. ${ }^{7}$ This information then needs to be integrated into the computer control system to allow for changes in position as the surgery progresses. This has been achieved using Direct Image Guided Intervention (DIGI) robots. These robots use pre-operative images to locate targets and create an initial navigation protocol. They are also connected to an imaging modality which allows for real-time adjustments to this protocol, providing greater accuracy and precision than a feed-forward system. Some challenges to DIGI include compatibility between robot and imaging modality such as MRI which has a dense magnetic field, and most components of the robotic system cannot be safely used in proximity to the magnet. Other problems include spatial resolution, and exposure to radiation if fluoroscopy or CT is used. ${ }^{8}$ The Robarts Research Institute at Western University is investigating the use of two-dimensional transrectal ultrasound (TRUS) to create three-dimensional real-time images by sweeping the probe about its axis. This technology has been combined with surgeon operated robotic surgery for urologic procedures such as prostate brachytherapy or biopsy. ${ }^{9}$

Another area of research with the potential to improve computer assisted surgery is haptics. Haptics is the general term used for touch feedback. This includes both tactile feedback (i.e. temperature, vibration, texture, etc) and kinaesthetic feedback (i.e. force and position). The majority of the work done regarding haptics in surgical robotics is surrounding kinaesthetic feedback, rather than tactile feedback. ${ }^{10} \mathrm{~A}$ known drawback of robot assisted minimally invasive surgery using such surgical robots is the lack of haptic feedback. ${ }^{11-13}$ This drawback has been found to be most limiting during complex tasks and can lead to difficulty identifying tissue based on consistency (i.e. between normal and tumour tissue) and suture breakage during knot tying. ${ }^{14-18}$

The reason for the lack of haptic feedback in such systems is simple: the technology required is incredibly complex. The problem of providing haptic feedback can be broken down into two distinct tasks: measuring the interaction forces and displaying these forces to the surgeon. Measuring of interaction forces during robotic surgery has been attempted in a few different manners. Retrofitting existing surgical manipulators with commercially available force sensors has been attempted ${ }^{19}$ however, constraints regarding size, geometry, biocompatibility, sterilizability and cost have limited this approach. ${ }^{10}$ Another method is to use the distance between desired position and actual position of the manipulator to determine force interactions between the manipulator and the environment. This method has been implemented with some success; however, it is limited by the non-ideal nature of the robots and the environment, meaning that friction and other dynamic forces are difficult to account for and can be large in comparison to the interaction forces between the manipulator and the patient. ${ }^{10,20}$

Methods of providing feedback to the surgeon include direct force feedback where motors in the robot controls are programmed to recreate the forces sensed by the manipulator, and the use of other sensory 


\section{MEDICINE AND TECHNOLOGY}

feedback such as audio or visual feedback to represent the forces experienced by the surgical instrument. The first method is the most commonly implemented method; ${ }^{10}$ however, enabling a system to provide force feedback in all directions actuated by the system (i.e. six degrees of freedom plus gripping) is not possible without implementing a second feedback system. ${ }^{22}$ Use of motors to provide feedback also introduces the possibility of feedback instability which can lead to uncontrolled oscillations, an obviously undesirable event during surgery. ${ }^{10}$ To get around these problems, various groups have attempted to implement feedback systems using visual or audio displays of force. ${ }^{14,23-25}$ These systems have experienced some success and have potential for further implementation as long as they do not interfere with the surgeon's view of the tissue through the video display of the endoscopic camera. ${ }^{10}$

Robotic surgery allows precise and repeated motions with decreased fatigue, smoother actions and increased accuracy, flexibility, and range of motion, especially in small confined anatomical locations such as the male pelvis during prostate surgery. However, there are still limitations in visual and haptic feedback. These limitations, as well as training of surgeons, have stunted the growth of this field in some ways; however, as described, intensive research is underway. It is clear that in certain situations, it is best to rely on image guidance, allowing the surgeon to be guided by more than what is visible through the visual field. For this to happen, imaging modalities which allow for greater compatibility with the surgical robot while maintaining as low radiation dose to the patient and surgeon as possible will need to be developed beyond the current technology. Similarly, to better enable this physician to identify specific tissues, haptic technology and its implementation into surgery will also need to be furthered. As these technologies become increasingly incorporated in the field of surgical robotics, we can expect rapid growth in the number of surgeries to make the transition from traditional to robotically-assisted or even fully automated.

\section{REFERENCES}

1. Kumar R, Hemal AK. Emerging role of robotics in urology. J Minim Access Surg. 2005 Oct; 1(4): 202-210.

2. Ahlering TE, Woo D, Eichel L, Lee DI, Edwards R, Skarecky DW. Robotassisted versus open radical prostatectomy: a comparison of one surgeon's outcomes. J Urol. 2004 May; 63(5): 819-822.

3. Patel VR, Chammas MF, Shah S. Robotic assisted laparoscopic radical prostatectomy: a review of the current state of affairs. Int J Clin Pract. 2007 Feb; 61(2): 309-314.

4. Moustris GP, Hiridis SC, Deliparaschos KM, Konstantinidis KM. Evolution of autonomous and semi-autonomous robotic surgical systems: a review of the literature. Int J Med Robot Comp. 2011 Dec; 7(4): 375-392.

5. Kimura T, Sakuramachi S, Yoshida M, Kobayashi T, Takeuchi Y. Laparoscopic cholecystectomy using fine-caliber instruments. Surg Endosc. 1998 Mar; 12(3): 283-286.

6. Lee C, Wang YF, Uecker DR, Wang Y. Image analysis for automated tracking in robot-assisted endoscopic surgery. Comput Vis Image Proc. 1994: 88-92.

7. Riener R, Rasmus W, Feussner. Acquisition of arm and instrument movements during laparoscopic interventions. Minim Invasive Ther Allied Technol. 2003 Sep; 12(5): 235-240.

8. Wei Z, Wan G, Gardi L, Mills G, Downey D, Fenster A. Robot-assisted 3DTRUS guided prostate brachytherapy: system integration and validation. Med Phys. 2004 Mar; 31(3): 539-548.

9. Dimaio S, Kapur T, Cleary K, Aylward S, Kazanzides P, Vosburgh K, Ellis R, Duncan J, Farahani K, Lemke H, Peters T, Lorensen WB, Gobbi D, Haller J, Clarke LL, Pizer S, Taylor R, Galloway R Jr, Fichtinger G, Hata N, Lawson $\mathrm{K}$, Tempany C, Kikinis R, Jolesz F. Challenges in image-guided therapy system design. Neuroimage. 2007 Apr; 37.

10. Okamura AM. Haptic feedback in robot-assisted minimally invasive surgery.
Curr Opin Urol. 2009; 19(1): 102-107.

11. Vivekananda U, Henderson A, Murphy DG, Althoefer L, Seneviratne L, Dasgupta $\mathrm{P}$. The science behind haptics in robotic urological surgery. BJU Int. 2009 Aug; 104(4): 433-434.

12. Bark K, McMahan W, Remington A, Gewirtz J, Wedmid A, Lee DI, Kuchenbecker KJ. In vivo validation of a system for haptic feedback of tool vibrations in robotic surgery. Surg Endosc. 2012 Jul.

13. Freschi C, Ferrari V, Melfi F, Ferrari M, Mosca F, Cuschieri A. Technical review of the da Vinci surgical telemanipulator. Int J Med Robot. 2012.

14. Kitagawa M, Dokko D, Okamura AM, Yuh DD. Effect of sensory substitution on suture-manipulation forces for robotic surgical systems. J Thorac Cardiovasc Surg. 2005 Jan; 129(1): 151-158.

15. Tholey G, Desai JP, Castellanos AE. Force feedback plays a significant role in minimally invasive surgery: results and analysis. Ann Surg. 2005 Jan; 241(1): 102-109.

16. Judkins TN, Oleynikov D, Stergiou N. Real-time augmented feedback benefits robotic laparoscopic training. Stud Health Technol Inform. 2006; 119: 243-248.

17. Diks J, Nio D, Linsen MA, Rauwerda JA, Wisselink W. Suture damage during robot-assisted vascular surgery: is it an issue? Surg Laparosc Endosc Percutan Tech. 2007 Dec;17(6): 524-527.

18. Ricchiuti D, Cerone J, Shie S, Jetley A, Noe D, Kovacik M. Diminished suture strength after robotic needle driver manipulation. J Endourol. 2010 Sep; 24(9): 1509-1513.

19. Dargahi J, Sedaghati R, Singh H, Najarian S. Modeling and testing of an endoscopic piezoelectric-based tactile sensor. J Mechatron. 2007; 17(8): $462-467$.

20. Mavash M, Okamura AM. Friction compensation for enhancing transparency of a teleoperator with compliant transmission. IEEE T Robotic Autom. 2007 23(6): 1240-1246.

21. Semere W, Kitagawa M, OkamuraAM. Teleoperation with Sensor/Actuator Asymmetry: Task Performance with Partial Force Feedback. 12th Symposium on Haptic Interfaces for Virtual Environments and Teleoperator Systems. 2004: 121-127.

22. Verner LN, Okamura AM. Effects of translational and gripping force feedback are decoupled in a 4-degree-of-freedom telemanipulator. Second Joint Eurohaptics Conference and Symposium on Haptic Interfaces for Virtual Environment and Teleoperator Systems (World Haptics). 2007: 286-291.

23. Okamura, A M. Methods for haptic feedback in teleoperated robot-assisted surgery. Ind Rob. 2004 Dec; 31(6): 499-508.

24. Schoonmaker RE, Caroline GL. Vibrotactile force feedback system for minimally invasive surgical procedures. IEEE Sys Man Cybern. 2006: 2464-2469.

25. Tavakoli M, Aziminejad A, Patel RV, Moallem M. Methods and mechanisms for contact feedback in a robot-assisted minimally invasive environment. Surg Endosc. 2006 Oct; 20(10): 1570-1579. 